\title{
Age at onset of mental disorders worldwide: large-scale meta- analysis of 192 epidemiological studies
}

\author{
Marco Solmi ${ }^{10,2,3}$ - Joaquim Radua ${ }^{3,4,5}$ - Miriam Olivola ${ }^{3}$ Enrico Croce ${ }^{6}$ - Livia Soardo $\mathbb{1}^{7}$.

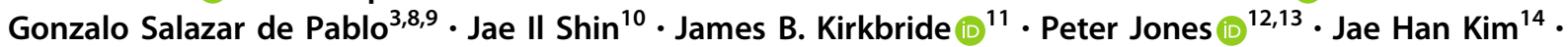 \\ Jong Yeob Kim ${ }^{14} \cdot$ Andrè F. Carvalho ${ }^{15} \cdot$ Mary V. Seeman $\mathbb{1}^{16} \cdot$ Christoph U. Correll $^{17,18,19,20}$. \\ Paolo Fusar-Poli $\mathbb{1}^{3,7,21,22}$
}

Received: 6 March 2021 / Revised: 28 April 2021 / Accepted: 5 May 2021 / Published online: 2 June 2021

(c) The Author(s) 2021. This article is published with open access

\begin{abstract}
Promotion of good mental health, prevention, and early intervention before/at the onset of mental disorders improve outcomes. However, the range and peak ages at onset for mental disorders are not fully established. To provide robust, global epidemiological estimates of age at onset for mental disorders, we conducted a PRISMA/MOOSE-compliant systematic review with meta-analysis of birth cohort/cross-sectional/cohort studies, representative of the general population, reporting age at onset for any ICD/DSMmental disorders, identified in PubMed/Web of Science (up to 16/05/2020) (PROSPERO:CRD42019143015). Co-primary outcomes were the proportion of individuals with onset of mental disorders before age 14, 18, 25, and peak age at onset, for any mental disorder and across International Classification of Diseases 11 diagnostic blocks. Median age at onset of specific disorders was additionally investigated. Across 192 studies $(n=708,561)$ included, the proportion of individuals with onset of any mental disorders before the ages of $14,18,25$ were $34.6 \%, 48.4 \%, 62.5 \%$, and peak age was 14.5 years $(k=14$, median $=18$, interquartile range $(\mathrm{IQR})=11-34)$. For diagnostic blocks, the proportion of individuals with onset of disorder before the age of $14,18,25$ and peak age were as follows: neurodevelopmental disorders: $61.5 \%, 83.2 \%, 95.8 \%, 5.5$ years $(k=21$, median $=12$, IQR $=7-16)$, anxiety/fear-related disorders: $38.1 \%, 51.8 \%, 73.3 \%, 5.5$ years $(k=73$, median $=17, \mathrm{IQR}=9-25)$, obsessive-compulsive/related disorders: $24.6 \%, 45.1 \%, 64.0 \%, 14.5$ years $(k=20$, median $=19, \mathrm{IQR}=14-29)$, feeding/eating disorders/problems: $15.8 \%$, $48.1 \%, 82.4 \%, 15.5$ years $(k=11$, median $=18, \mathrm{IQR}=15-23)$, conditions specifically associated with stress disorders: $16.9 \%$, $27.6 \%, 43.1 \%, 15.5$ years $(k=16$, median $=30, \mathrm{IQR}=17-48)$, substance use disorders/addictive behaviours: $2.9 \%, 15.2 \%$, $48.8 \%, 19.5$ years $(k=58$, median $=25, \mathrm{IQR}=20-41)$, schizophrenia-spectrum disorders/primary psychotic states: $3 \%, 12.3 \%$, $47.8 \%, 20.5$ years $(k=36$, median $=25, \mathrm{IQR}=20-34)$, personality disorders/related traits: $1.9 \%, 9.6 \%, 47.7 \%, 20.5$ years $(k=6$, median $=25, \mathrm{IQR}=20-33)$, and mood disorders: $2.5 \%, 11.5 \%, 34.5 \%, 20.5$ years $(k=79$, median $=31, \mathrm{IQR}=21-46)$. No significant difference emerged by sex, or definition of age of onset. Median age at onset for specific mental disorders mapped on a time continuum, from phobias/separation anxiety/autism spectrum disorder/attention deficit hyperactivity disorder/social anxiety (8-13 years) to anorexia nervosa/bulimia nervosa/obsessive-compulsive/binge eating/cannabis use disorders (17-22 years), followed by schizophrenia, personality, panic and alcohol use disorders (25-27 years), and finally post-traumatic/depressive/generalized anxiety/bipolar/acute and transient psychotic disorders (30-35 years), with overlap among groups and no significant clustering. These results inform the timing of good mental health promotion/preventive/early intervention, updating the current mental health system structured around a child/adult service schism at age 18 .
\end{abstract}

These authors contributed equally: Marco Solmi, Joaquim Radua

Supplementary information The online version contains supplementary material available at https://doi.org/10.1038/s41380021-01161-7.

Paolo Fusar-Poli

paolo.fusar-poli@kcl.ac.uk

Extended author information available on the last page of the article

\section{Introduction}

Individuals with mental disorders have a decreased life expectancy of 10-15 years in comparison with the general population [1-4]. Early interventions at the first onset of mental disorders can improve several outcomes [5, 6]. Primary indicated prevention in those at clinical high risk has the potential to alter the course of the disorder and 
improve outcomes [7-9]. For example, young people with attenuated symptoms for psychosis [10-13] and functional impairments accumulate several risk factors and have a $25 \%$ probability of developing the disorder over 3 years [14]. Clinical care for these individuals is typically implemented in specialised clinical services [15-18] and has the potential to delay or impede the transition to psychosis, although the efficacy of preventive interventions awaits more robust evidence [19-21]. Targeted preventive approaches involve screening programmes in asymptomatic individuals who have significant risk factors for certain psychiatric disorders $[7,22,23]$ (primary selective prevention [7, 8]) or public health campaigns in the general population (primary universal prevention) $[7,8,24]$. To date, these initiatives have been mostly piloted for young people with emerging severe mental disorders [8]. A further complementary approach is to promote good mental health, as opposed to preventing mental disorders [7, 25, 26].

Although promotion of good mental health, prevention and early intervention can be implemented over the lifespan, the benefits are maximal when young people are targeted at around the time of onset of mental disorders. Unfortunately, the peak ages and ranges at onset for mental disorders are not fully established, with conflicting findings across [27, 28] and within studies [29], partly due to methodological limitations, including selection biases in recruitment for clinical studies [30]. General populationlevel studies (birth cohort, cross-sectional or incidence studies) provide the most robust onset age estimates [30]. However, to date, no comprehensive epidemiologically sound, large-scale meta-analysis has pooled data from these population-based studies that are representative of the general population to estimate the peak age at the onset across the globe and the proportion of individuals with mental disorders at specific age points. This study's goal was to fill this gap aiming to optimise timely intervention, prevention and promotion of good mental health opportunities at the time of onset of mental disorders.

\section{Method}

\section{Search strategy}

A study protocol was registered and is publicly available on PROSPERO (CRD42019143015). We performed a systematic review adhering to the preferred reporting items for systematic reviews and meta-analyses (PRISMA) recommendations [31] (e-Table 1) and the meta-analysis of observational studies in epidemiology (MOOSE) guidelines (e-Table 2) [32].

At least two authors (MO, ER, LS, MS, all MDs) independently searched PubMed and Web of Science using the following terms: "age at onset" (topic) and "mental disorder" (topic), plus "birth cohort" (topic) and "mental disorder" (topic), plus "incidence" (topic) and "mental disorder" (topic), without restriction to the type of mental disorder. Additionally, reviews and reference lists of included studies were manually searched. The literature was searched from database inception until 16/05/2020. Hits were first screened at the title/abstract level, then full texts of the remaining articles were assessed, recording reasons for exclusion (as per the criteria noted below).

\section{Inclusion and exclusion criteria}

Included were: (i) original birth cohort or cross-sectional studies retrospectively assessing age at disorder onset, or prospective incidence studies [30], (ii) focusing on the general population, (iii) assessing age at onset of mental disorders, defined according to international classification diseases (ICD) or diagnostic and statistical manual (DSM)any version criteria, or by established psychometric instruments with validated cut-offs that defined ICD/DSM diagnostic categories, (iv) written in English.

Excluded were: (i) studies sampling clinical groups that were not representative of the general population, (ii) studies assessing the prevalence, not onset age of mental disorders, (iii) reviews, meta-analyses, case reports or other non-original studies, (iv) non-English language articles.

\section{Data extraction}

The following variables were extracted into pre-defined excel spreadsheets: DOI/PMID, author, publication year, country of study, study design (birth cohort, cross-sectional, incidence studies, all representative of the general population), name of the cohort (if available), ICD/DSM diagnostic criteria, onset definition (first symptoms, first diagnosis, first hospitalisation), a specific type of mental disorder, sample age range, number of participants, number of cases developing incident mental disorders. Age at onset of mental disorders was extracted as available in each of the included studies (see statistics). Data extraction was performed independently by the same pairs of authors who performed the literature screening.

\section{Study quality assessment}

To the best of our knowledge, no quality assessment measure has been validated for the type of studies included in the current meta-analysis. Therefore, the risk of bias was evaluated with an ad-hoc list of criteria derived from the Newcastle-Ottawa scale (NOS) [33], which included the definition of onset age of the mental disorder, diagnostic criteria employed, and study design. However, since these 
criteria were not validated, they were not employed to categorise studies according to their quality; they were only used for descriptive reporting.

\section{Statistical analysis}

Study-defined age at onset of mental disorders encompassed: (i) mean and standard deviation (SD), percentiles, median and/or interquartile range (IQR) of age at disorder onset; (ii) proportion of cases of a sample whose age at disorder onset fell into a certain age group, (iii) number of incident cases developing mental disorders, separately for age groups (e.g., for national registries).

We defined co-primary outcomes as the proportion of individuals with age at disorder onset of any and specific mental disorder groups before 14, 18 and 25 years old, and peak age at onset for any mental disorder and for each diagnostic group. Diagnostic groups matched all 19 ICD-11 diagnostic blocks under "Mental, behavioural or neurodevelopmental disorder", namely neurodevelopmental disorders, schizophrenia-spectrum and primary psychotic disorders, catatonia, mood disorders, anxiety and fearrelated disorders, obsessive-compulsive or related disorders, disorders specifically associated with stress, dissociative disorders, feeding or eating disorders, elimination disorders, disorders of bodily experience, disorders due to substance use or addictive behaviour, impulse-control disorders, disruptive behaviour or dissocial disorders, personality disorders and related traits, paraphilic disorders, factitious disorders, neurocognitive disorders, disorders associated with pregnancy childbirth or puerperium. Individual studies adopted different age subgroupings and age ranges. To our knowledge, there is no standard method to pool such varying descriptive statistics of the distribution of a variable (age at onset of mental disorders) when the variable of interest follows a non-normal distribution and/or is heterogeneously censored. Therefore, we have developed an adhoc method to meta-analyse these estimates. To assess these co-primary outcomes, we first estimated the histogram of the age at disorder onset that minimised the sum of squared errors (SSE) of the study-reported data. From this histogram, we then derived peak age at onset, as well as the proportion of individuals showing an onset before 14, 18 and 25 years of age, and the $25 \%, 50 \%$ and $75 \%$ percentiles of the age at disorder onset. Thereafter, we used an empirical bootstrap approach [34] to estimate the $95 \%$ confidence intervals of age at onset.

Additional sensitivity analyses were performed. First, we repeated the analyses for specific mental disorders, preselected on the basis of their epidemiological and clinical relevance: attention-deficit/hyperactivity disorder (ADHD), autism spectrum disorder (ASD), generalised anxiety disorder (GAD), panic disorder, separation anxiety disorder, specific phobia, social anxiety disorder, obsessivecompulsive disorder (OCD), disorders due to use of alcohol, disorders due to use of cannabis, anorexia nervosa, bulimia nervosa, binge eating disorder, depressive disorders, bipolar or related disorders (bipolar disorder), acute and transient psychotic disorders (ATPD), schizophrenia, and post-traumatic stress disorder (PTSD). Second, we repeated analyses for any disorder, stratifying by onset definition (i.e., first symptoms, first diagnosis, first hospitalisation). Third, we repeated the analyses for any disorder stratifying by sex. Finally, we compared the median age at onset between each pair of specific mental disorders, counting the proportion of bootstrapped randomisations in which the median age at onset of one disorder was larger than the median age of the other disorder (alpha $=0.05$, two-sided).

For all analyses, we required $\geq 2$ studies to estimate the histogram and to apply the bootstrap procedure. For full details regarding the statistical analysis, see e-methods.

\section{Results}

\section{Literature search and database}

We identified 5,442 possible publications after removing duplicates, of which 4,516 were excluded after title/abstract screening. An additional 734 publications were excluded after full-text review (see e-Fig. 1 and supplementary material-e-Table 3-for full details). For characteristics of the 192 included studies (birth cohort studies $=15$, crosssectional studies $=150$, incidence studies $=27$ ), see eTable 4. These studies were comprised of data from 708,561 individuals, all of whom were diagnosed with a mental disorder. Overall, 54 studies were set in U.S., 23 studies in multiple countries, 11 in Australia, ten in Finland, eight in Germany, six each in Canada and the Netherlands, five in China, Denmark, South Africa, Spain, United Kingdom, four in Israel, South Korea, Sweden, three in Ethiopia, Mexico, New Zealand, Nigeria, Switzerland, Taiwan, two in France, Iraq, Singapore, and one study each in additional countries.

There were insufficient studies for inclusion on catatonia, dissociative disorders, elimination disorders, disorders of bodily experience, impulse-control disorders, disruptive behaviour or dissocial disorders, paraphilic disorders, factitious disorders, neurocognitive disorders, and disorders associated with pregnancy childbirth/puerperium.

\section{Global age at the onset across diagnostic spectra of mental disorders}

The proportion of individuals with age at onset before 14, 18 and 25 years of age and peak age at onset for diagnostic 


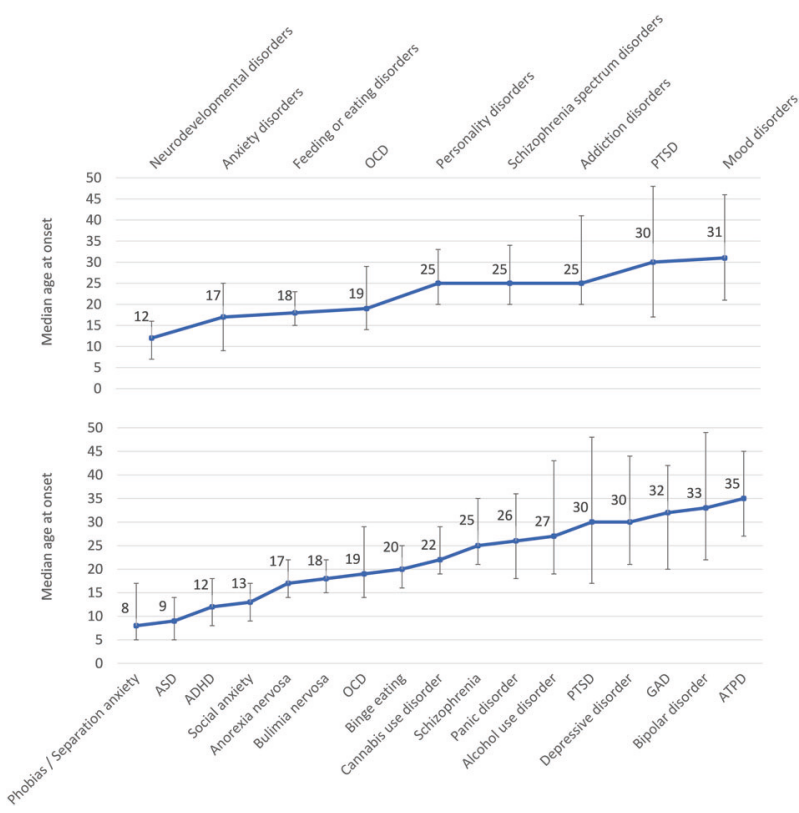

Fig. 1 Meta-analytic median age at onset of mental disorders. The line indicates the median age at onset of mental disorders (ICD-11 diagnostic blocks or spectra above, specific mental disorders below), the bar indicates the 25 th and 75 th percentiles. ICD-11 blocks of mental disorders. Addiction disorders: disorders due to substance use or addictive behaviour, Anxiety and fear: anxiety and fear-related disorders, OCD related: obsessive-compulsive or related disorders, Schizophrenia spectrum disorders: schizophrenia-spectrum and primary psychotic disorders. ICD-11 specific mental disorders. ADHD attention deficit hyperactivity disorder, ASD: autism spectrum disorder, ATPD, acute and transient psychotic disorder; Binge eating: binge eating disorder, Bipolar disorder: bipolar or related disorders, GAD generalised anxiety disorder, OCD obsessive-compulsive disorder, Phobia: specific phobia, PTSD post-traumatic stress disorder, Separation anxiety: separation anxiety disorder, Social anxiety: social anxiety disorder.

spectra (co-primary outcome measures) are reported in Table 1. Overall, before age 14, 18, and 25 years, a disorder had already emerged in $34.6 \%, 48.4 \%$, and $62.5 \%$ of individuals (Table 1).

Corresponding figures were, respectively, for neurodevelopmental disorders: $61.5 \%, 83.2 \%, 95.8 \%$, for anxiety/ fear-related disorders: $38.1 \%, 51.8 \%, 73.3 \%$, for obsessivecompulsive/related disorders: $24.6 \%, 45.1 \%, 64.0 \%$, for feeding/eating disorders: $15.8 \%, 48.1 \%, 82.4 \%$, for disorders specifically associated with stress: $16.9 \%, 27.6 \%$, $43.1 \%$, for substance use/addictive behaviour disorders: $2.9 \%, 15.2 \%, 48.8 \%$, and for schizophrenia-spectrum/primary psychotic disorders: $3 \%, 12.3 \%, 47.8 \%$, for personality disorders/traits: $1.9 \%, 9.6 \%, 47.7 \%$, and for $\operatorname{mood}$ disorders: $2.5 \%, 11.5 \%, 34.5 \%$.

Curves representing the median, 25th, and 75th percentiles and peak age at onset for mental disorder spectra are reported in Figs. 1-4. The peak and median age at onset for any mental disorder were 14.5 years and 18 years. The earliest age peaks of disorder onset were: neurodevelopmental disorders $\quad($ peak $=5.5 /$ median $=12$ years), anxiety/fear-related disorders (peak $=5.5 /$ median $=$ 17 years), obsessive-compulsive/related disorders (peak = $14.5 /$ median $=18$ years $)$, feeding/eating disorders $($ peak $=$ $15.5 /$ median $=18$ years , disorders specifically associated with stress (peak $=15.5 /$ median $=30$ years), substance use/ addictive behaviour disorders $($ peak $=19.5 /$ median $=25$ years), schizophrenia-spectrum/primary psychotic and personality disorders/traits (peak $=20.5 /$ median $=25$ years), and mood disorders (peak $=20.5 /$ median $=31$ years). Second age peaks emerged for any mental disorder at (peak $=30.5$ years), anxiety/fear-related disorders (peak $=15.5$ years), obsessive-compulsive/related disorders (peak $=49.5$ years), disorders specifically associated with stress (peaks $=30.5$ and 49.5 years), and substance use/addictive behaviour disorders (peak $=44.5$ years).

\section{Sensitivity analyses}

The proportion of individuals with age at the onset before 14, 18 and 25 years of age for specific mental disorders are reported in Table 1. Overall, the proportion of patients developing disorders before age 25 is more than nine out of ten for ASD and ADHD; nine out of ten for social anxiety disorder; around eight out of ten for specific phobia/ separation anxiety disorders and anorexia nervosa; seven to eight out of ten for binge eating disorders; six to seven out of ten for OCD and disorders due to use of cannabis; four to five out of ten for disorders due to use of alcohol, schizophrenia, PTSD, panic disorder, and personality disorders; three to four out of ten for GAD, bipolar/related disorders, and depressive disorders; and two out of ten for ATPD. The curves representing the peak age at onset for specific mental disorders are presented in e-Figs. 2-17.

As reported in Table 2, the age at onset was mostly similar between males and females, although there was a trend towards younger ages for males in disorders due to substance use or addictive behaviours (median $=4$ years earlier), mood disorders (median $=2$ years earlier) or schizophrenia-spectrum and primary psychotic disorders (median $=1$ year earlier). Similarly, there were little differences in age at onset among studies where onset was defined according to first symptoms, first diagnosis or first hospitalisation, although there was a trend towards younger ages for first symptoms than for the first diagnosis in disorders due to substance use/addictive behaviours (median symptoms 9 years earlier), mood disorders (median 8 years earlier), anxiety and fear-related disorders (median 3 years earlier). For schizophrenia-spectrum and primary psychotic disorders, we observed a trend from symptoms to hospital admission (median a year later) to diagnosis (median another year later). There were other minor differences in disorders with fewer $(<10)$ separate studies for sex or onset 
Table 1 Meta-analytic epidemiological estimates of age at onset of mental disorder (ICD-11 blocks highlighted in italics) in the general population.

\begin{tabular}{|c|c|c|c|c|c|c|c|c|}
\hline & $\begin{array}{l}\text { No of } \\
\text { Samples }\end{array}$ & $\begin{array}{l}\text { Peak age at } \\
\text { onset (years) }\end{array}$ & $\begin{array}{l}\text { Proportion onset } \\
\text { by } 14 \text { years }\end{array}$ & $\begin{array}{l}\text { Proportion onset } \\
\text { by } 18 \text { years }\end{array}$ & $\begin{array}{l}\text { Proportion onset } \\
\text { by } 25 \text { years }\end{array}$ & $\mathrm{p} 25$ & Median & $\mathrm{p} 75$ \\
\hline Any mental disorder & 14 & 14.5 & $34.6 \%$ & $48.4 \%$ & $62.5 \%$ & 11 & 18 & 34 \\
\hline Neurodevelopmental disorders & 21 & 5.5 & $61.5 \%$ & $83.2 \%$ & $95.8 \%$ & 7 & 12 & 16 \\
\hline Autism spectrum disorder & 2 & 5.5 & $72.4 \%$ & $89.8 \%$ & $94.8 \%$ & 5 & 9 & 14 \\
\hline $\begin{array}{l}\text { Attention deficit hyperactivity } \\
\text { disorder }\end{array}$ & 12 & 9.5 & $56.8 \%$ & $73.0 \%$ & $91.8 \%$ & 8 & 12 & 18 \\
\hline Anxiety and fear-related disorders & 73 & 5.5 & $38.1 \%$ & $51.8 \%$ & $73.3 \%$ & 9 & 17 & 25 \\
\hline $\begin{array}{l}\text { Specific phobia/separation anxiety } \\
\text { disorder }\end{array}$ & 22 & 5.5 & $72.4 \%$ & $75.0 \%$ & $80.4 \%$ & 5 & 8 & 17 \\
\hline Social anxiety disorder & 42 & 14.5 & $50.9 \%$ & $79.1 \%$ & $87.5 \%$ & 9 & 13 & 17 \\
\hline Panic disorder & 22 & 15.5 & $8.2 \%$ & $22.5 \%$ & $45.7 \%$ & 18 & 26 & 36 \\
\hline Generalised anxiety disorder & 24 & 15.5 & $8.6 \%$ & $20.4 \%$ & $33.0 \%$ & 20 & 32 & 42 \\
\hline $\begin{array}{l}\text { Obsessive-compulsive related } \\
\text { disorders }\end{array}$ & 20 & 14.5 & $24.6 \%$ & $45.1 \%$ & $64.0 \%$ & 14 & 19 & 29 \\
\hline Obsessive-compulsive disorder & 20 & 14.5 & $24.6 \%$ & $45.1 \%$ & $64.0 \%$ & 14 & 19 & 29 \\
\hline Feeding or eating disorders & 11 & 15.5 & $15.8 \%$ & $48.1 \%$ & $82.4 \%$ & 15 & 18 & 23 \\
\hline Anorexia nervosa & 8 & 15.5 & $18.2 \%$ & $55.2 \%$ & $78.7 \%$ & 14 & 17 & 22 \\
\hline Bulimia Nervosa & 8 & 15.5 & $16.0 \%$ & $45.3 \%$ & $82.9 \%$ & 15 & 18 & 22 \\
\hline Binge eating disorder & 5 & 19.5 & $12.3 \%$ & $34.5 \%$ & $73.5 \%$ & 16 & 20 & 25 \\
\hline Trauma-related disorders & 16 & 15.5 & $16.9 \%$ & $27.6 \%$ & $43.1 \%$ & 17 & 30 & 48 \\
\hline Post-traumatic stress disorder & 16 & 15.5 & $16.9 \%$ & $27.6 \%$ & $43.1 \%$ & 17 & 30 & 48 \\
\hline $\begin{array}{l}\text { Disorders due to substance use or } \\
\text { addictive behaviours }\end{array}$ & 58 & 19.5 & $2.9 \%$ & $15.2 \%$ & $48.8 \%$ & 20 & 25 & 41 \\
\hline Disorder due to use of cannabis & 10 & 19.5 & $3.2 \%$ & $17.5 \%$ & $64.6 \%$ & 19 & 22 & 29 \\
\hline Disorder due to use of alcohol & 44 & 19.5 & $4.2 \%$ & $18.3 \%$ & $44.8 \%$ & 19 & 27 & 43 \\
\hline $\begin{array}{l}\text { Schizophrenia-spectrum and } \\
\text { primary psychotic disorders }\end{array}$ & 36 & 20.5 & $3.0 \%$ & $12.3 \%$ & $47.8 \%$ & 20 & 25 & 34 \\
\hline Schizophrenia & 25 & 20.5 & $2.0 \%$ & $8.2 \%$ & $47.4 \%$ & 21 & 25 & 35 \\
\hline $\begin{array}{l}\text { Acute and transient psychotic } \\
\text { disorder }\end{array}$ & 2 & 18.5 & $1.8 \%$ & $6.6 \%$ & $20.6 \%$ & 27 & 35 & 45 \\
\hline Personality disorders & 6 & 20.5 & $1.9 \%$ & $9.6 \%$ & $47.7 \%$ & 20 & 25 & 33 \\
\hline Mood disorders & 79 & 20.5 & $2.5 \%$ & $11.5 \%$ & $34.5 \%$ & 21 & 31 & 46 \\
\hline Depressive disorders & 62 & 19.5 & $3.1 \%$ & $13.2 \%$ & $36.9 \%$ & 21 & 30 & 44 \\
\hline Bipolar or related disorders & 40 & 19.5 & $5.1 \%$ & $13.7 \%$ & $32.0 \%$ & 22 & 33 & 49 \\
\hline
\end{tabular}

p25, p75, 25th and 75th percentile. Only the earliest peaks are shown. Different metric on the same sample has been extracted from multiple studies.

definition, for which we suggest caution as the estimations may be less accurate than others.

Despite some differences shown in e-Table 5, the median age at onset of specific mental disorders maps on a continuum, with no clear clustering across different disorders. Descriptively, the earliest median age of onset was observed for phobias/separation anxiety, ASD, ADHD, social anxiety disorders (8-13 years), followed by anorexia nervosa, bulimia nervosa, OCD, binge eating, cannabis use disorders (17-22 years), later schizophrenia, personality and alcohol use disorders (25-27 years), and, finally, PTSD, depressive disorder, GAD, bipolar disorder, and ATPD (30-35 years).

\section{Discussion}

This is the first large-scale epidemiological meta-analysis that includes all available general population birth, crosssectional and incidence studies investigating the age at onset of any ICD/DSM-mental disorders. Overall, the global onset of the first mental disorder occurs before age 14 in one-third of individuals, age 18 in almost half (48.4\%), and before age 25 in half $(62.5 \%)$, with a peak/median age at onset of 14.5/18 years across all mental disorders. However, there was significant variability in global age at onset and peak age across mental disorders. These findings can inform 


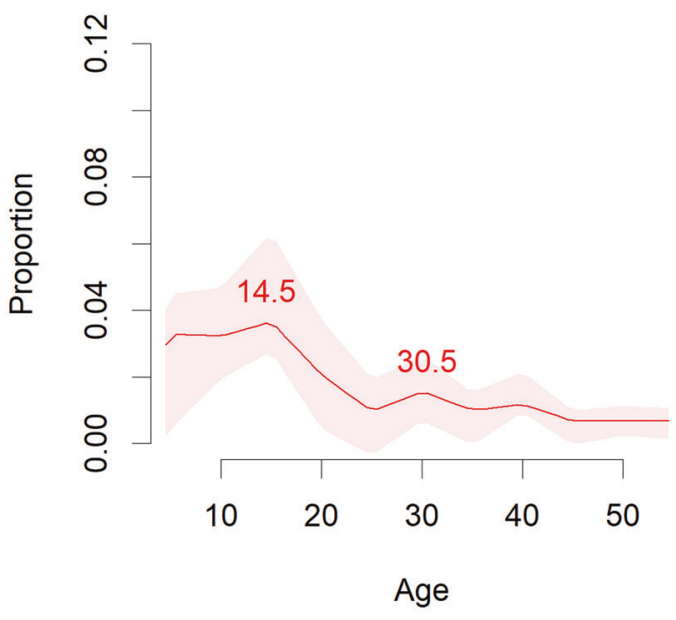

Fig. 2 Meta-analytic distribution of age of onset for any mental disorders. Meta-analytic epidemiological proportion ( $y$-axis) and peak age at onset (red line) for any mental disorders in the general population, with $95 \%$ CIs (pink shadows).

the timing and resource allocation regarding early intervention and preventive approaches.

To our best knowledge, this study is the first fully epidemiological and largest [35-38] meta-analysis on age at onset of mental disorders globally. It also represents the most comprehensive approach, encompassing all ICD-11 diagnostic spectra for which we found eligible studies, allowing comparative transdiagnostic analyses across different categories of mental disorders [39, 40]. Furthermore, per protocol, high-quality population-level studies were included that are less likely to be affected by biases, meeting previous methodological recommendations [30]. Moreover, data from all continents of the world were available, providing global estimates on the age at onset of mental disorders. Importantly, the statistical approach of this meta-analysis provides an estimate of age at disorder onset distribution throughout the lifespan, going beyond mere centrality estimates.

The meta-epidemiologic results of this work show that mental disorders have onset when dramatic biological changes in the brain occur, from childhood, through adolescence, to adulthood, that involves grey-matter density, cerebral metabolic rate, synaptic density, white matter growth and myelination [41]. The in-depth, robust epidemiological evidence provided here has several clinical implications. Firstly, the onset of the first mental disorder before age 14, 18 and 25 in one third, half and $62.5 \%$ and peak/median age of $15.5 / 18$ years demonstrate that adult mental disorders originate early during the neurodevelopmental phases of life and peak, in pooled mental disorders, by mid to late adolescence [42-44]. Given that mental disorders are one of the five most common ailments leading to morbidity, mortality and dysfunction among young people worldwide [45], the current findings are relevant to policymakers and healthcare providers. These results suggest that the next generation of mental health research should prioritise designing and funding global early interventions $[5,46]$ and indicated, selective and/or universal preventive interventions for mental disorders during mid/ late adolescence and young adulthood that are currently lacking [8].

Secondly, this study provides disorder-specific estimates of age at disorder onset that impacts mental health service configuration and delivery. For about half of mental disorders (Table 2), disorder onset occurs well before age 18 . Disorder median age at onset occurred during the neurodevelopment, within age 14 for the vast majority of phobias and separation anxiety, ASD, ADHD, and for more then half in social anxiety disorders. For these disorders, good mental health promotion, along with preventive and early intervention approaches need to target these neurodevelopmental periods, mainly during pre-school and primary school periods. Many of the risk and protective factors that impact the neurodevelopment of individuals with these disorders are known [23, 47-52], and some of them may even impact the pre or perinatal phases [53]. A recent metaumbrella review (i.e., a synthesis of systematic reviews of meta-analyses) has summarised risk and protective factors (beyond genetics) in a comprehensive atlas [54]. Importantly, targeting the neurodevelopmental phase has the potential to accommodate multi-endpoint numerators across mental disorders that are essential to better justify the denominator of efforts and costs for preventive and early intervention [8]. Higher median age at disorder onset during the transitional period across adolescence and young adulthood emerged for another larger group of mental disorders, i.e., anorexia nervosa, bulimia nervosa, OCD, binge eating, and cannabis use disorders. For these disorders, good mental health promotion, prevention and early intervention could be delivered in primary and secondary schools [55-57]. A third group with median age at onset in the early adulthood included, schizophrenia, personality, panic and alcohol use disorders, and a fourth group included the remaining disorders that have a median age at onset in the later adulthood, such as PTSD, GAD, depressive and bipolar disorders, and ATPD. Secondary schools and colleges could become the most important setting for mental health promotion, prevention and early intervention across these two groups of mental disorders. Importantly, the diagnosis of personality disorder is artifactually delayed by diagnostic criteria allowing diagnosis after age 18, and clinically relevant symptoms occur earlier.

For all disorders culturally and ethically- sensitive [8] promotion of good mental health (indicated, selective and/or universal) prevention and early intervention should ideally be delivered in an integrated fashion that encompasses schools/colleges, and paediatricians/general 
Neurodevelopmental disorders

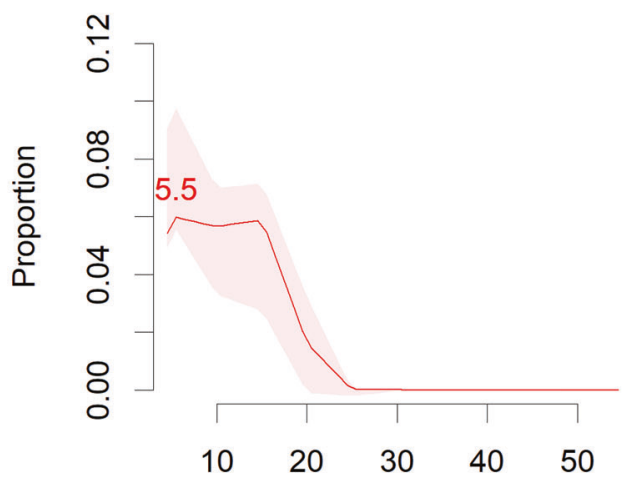

Age
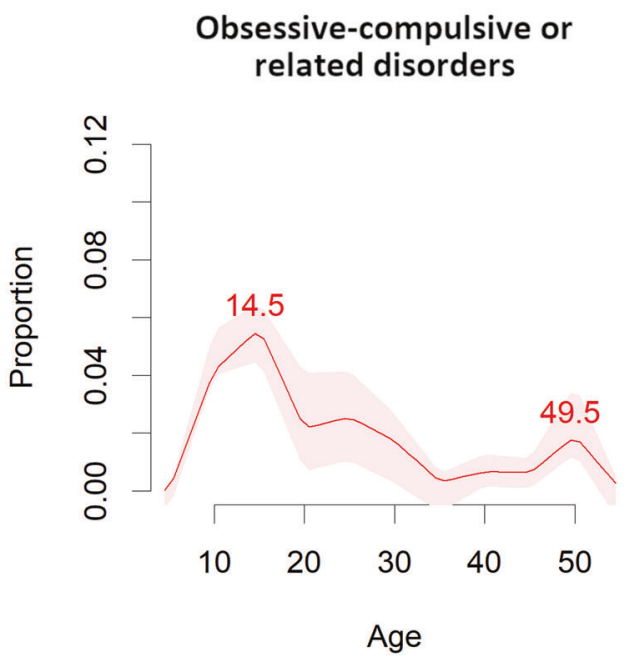

Anxiety and fear related disorders

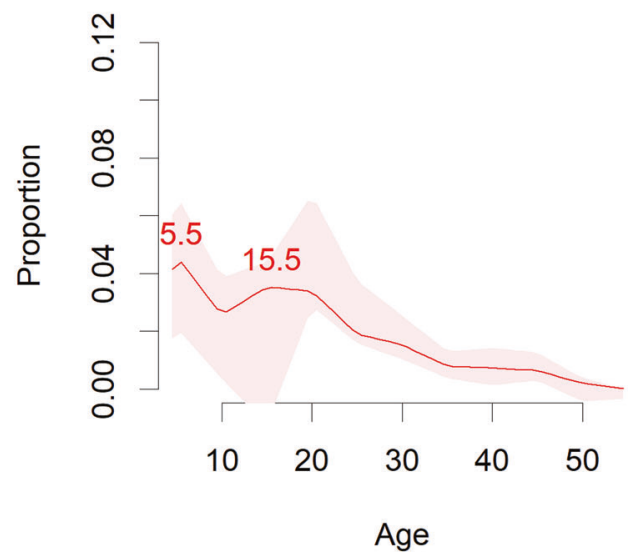

Feeding or eating disorders

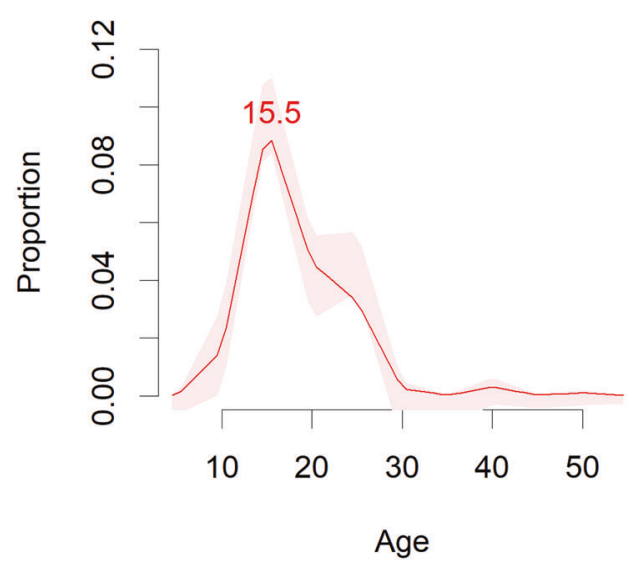

Fig. 3 Meta-analytic distribution of age of onset for specific mental disorders blocks. Meta-analytic epidemiological proportion ( $y$-axis) and peak age at onset (red line) for neurodevelopmental, anxiety and fear-related, obsessive-compulsive related, and feeding or eating disorders (ICD11 blocks) in the general population, with $95 \%$ CIs (pink shadows).

practitioners, emergency departments, mental health settings, as well as the general community [60]. Overall, this study shows that any lower age threshold limiting access to mental health promotion campaigns or preventive or early interventions mental health programmes is not supported by meta-epidemiological evidence. Conversely, lower age thresholds that divide the education and training of mental health specialists or clinical services deprive individuals with developmental disorders (or other disorders with early onset) of continuity of care. Divisions fragment pathways to care and continued treatment from childhood through adolescence into adulthood (see below) [58]. Our recommendation for mental health services of the future would allow soft entry points, set no lower age threshold, and loosen lower age intake criteria [59]. For example, an indicated prevention and early intervention model of care for mood disorders, and schizophrenia-spectrum/primary psychotic disorders should ideally integrate low-threshold entry points for individuals of age younger than 18, supplemented by systematic promotion of good mental health, proactive screening for risk of developing specific mental disorders according to peak and median age at onset identified above, and deliver needs-based care [19, 60, 61].

Thirdly, a further, broader clinical implication of these results is that it demonstrates that age 18 as an intake threshold for adult mental health services is artificial and not based on global epidemiological evidence (nor on biological evidence of age when major brain changes occur [41]). To the best of our knowledge, mental health specialist training and mental health services in most parts of the world, including North America, Australia, most European countries, including Italy, Germany, UK, are divided between child/adolescent psychiatry and adult psychiatry. Given that the vast majority of mental disorders diagnoses 

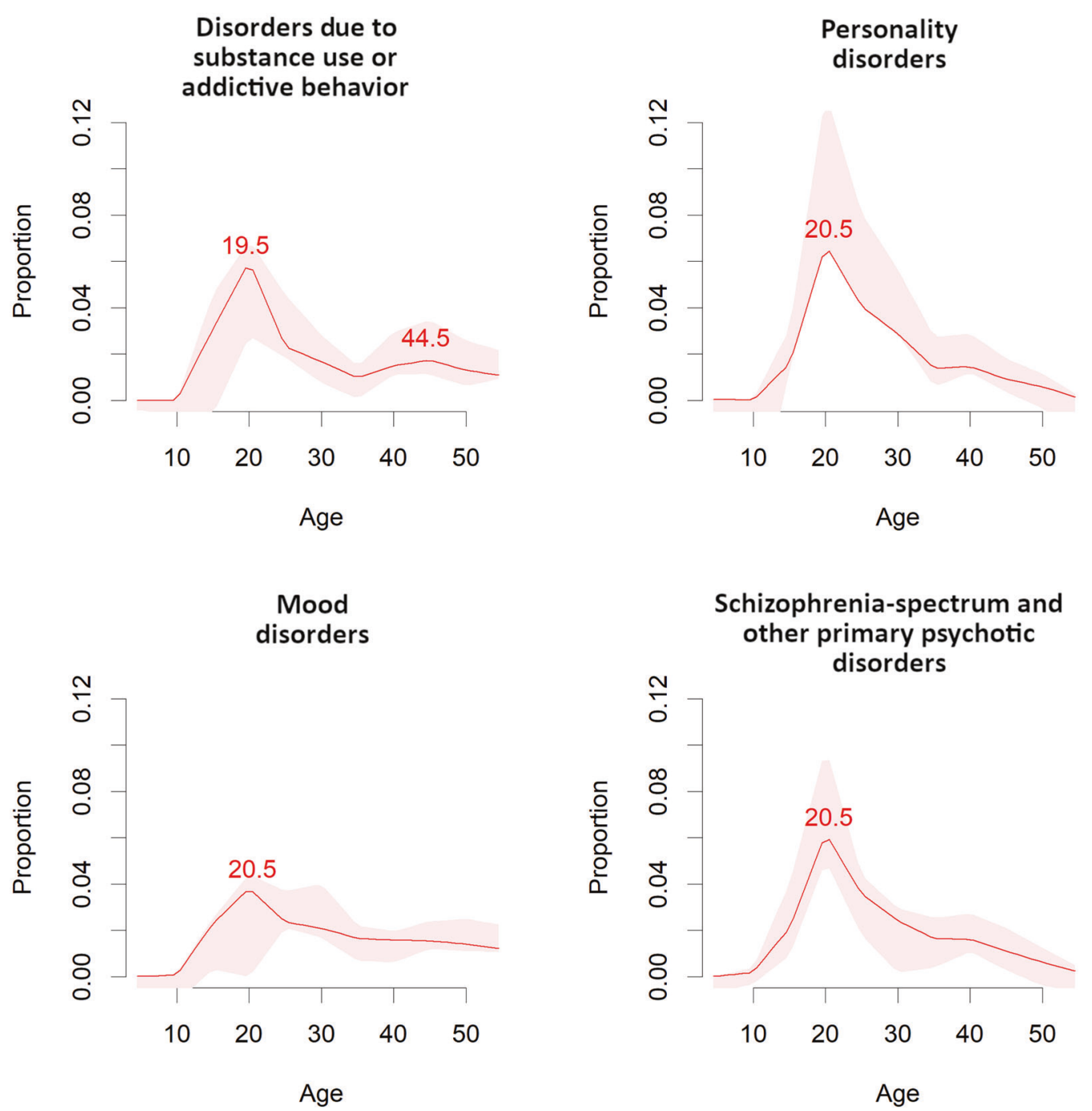

Fig. 4 Meta-analytic distribution of age of onset for specific mental disorders blocks. Meta-analytic epidemiological proportion ( $y$-axis) and peak age at onset (red line) for disorders due to substance use or addictive behaviour, personality, mood, and schizophrenia-spectrum and primary psychotic disorders (ICD-11 blocks) in the general population, with 95\%CIs (pink shadows).

seen in adulthood show a peak of onset before age 18, such a bifurcated mental health system is not evidence-based and may disadvantage individuals with developmental disorders from accessing and receiving adequate and continuous care [62]. Moreover, most psychiatry training programmes fail to target the transition period from childhood and adolescence to adult psychiatry [63]. Although many mental health services have tried to address this discontinuity of care [63, 64], significant gaps remain. Future mental health reforms could leverage and refine clinical high-risk services for young people at risk of psychosis, which typically accepts referrals aged 14-35 years and therefore provide essential transitional care to this vulnerable young population $[15,17,58]$.

This study has several limitations. First, data were too sparse to calculate country-specific estimates. However, the database included many countries worldwide, and, therefore, the estimates provided are largely representative of the global general population. Second, the 95\% confidence interval for some estimates were broad since analyses for some disorders were based on few studies. Third, data were heterogeneous, making traditional meta-analytic techniques unfeasible. We, therefore, applied advanced meta-analytic techniques, including bootstrap methods that helped to address this shortcoming and provide unified outcomes across all mental disorders. Fourth, our quality assessment was non-standardised because appropriate validated measures were not available. However, we perprotocol included the highest-quality studies to study age at onset according to previous recommendations [30]. Fifth, the definition of age at disorder onset was heterogeneous; we addressed this issue via sensitivity analyses. Sixth, we were not able to account for and differentiate among comorbid and standalone diagnoses. Sixth, we were not able 


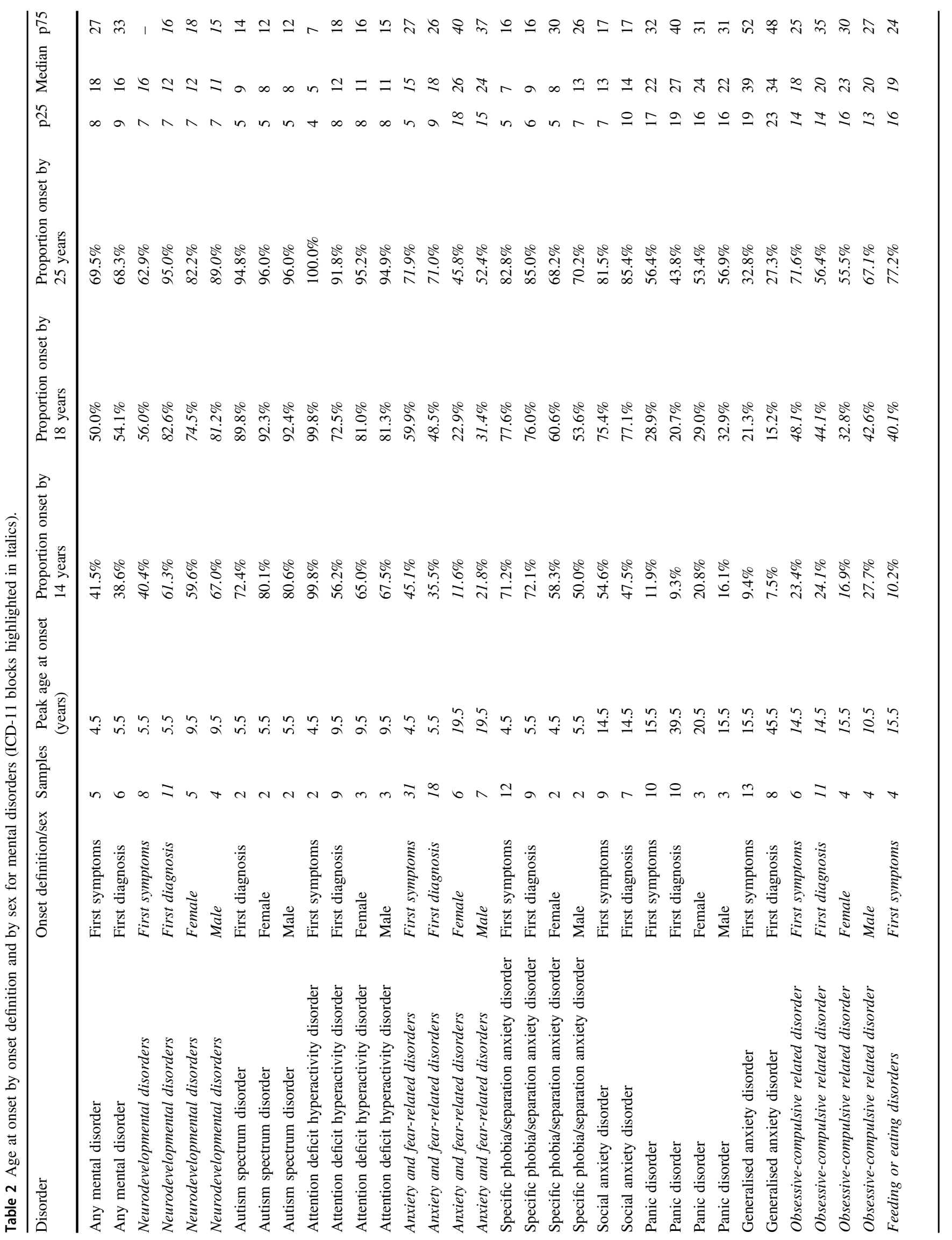




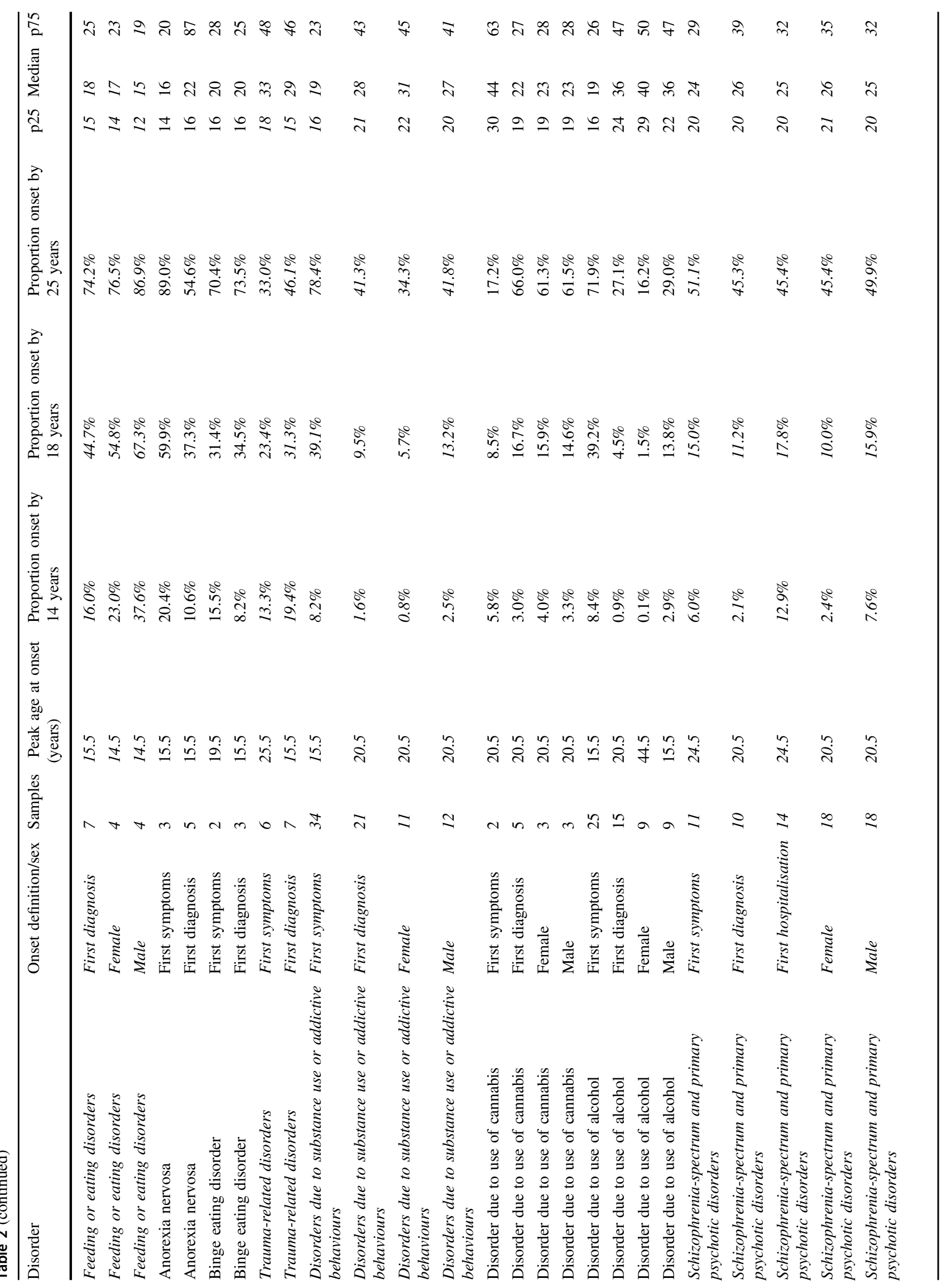




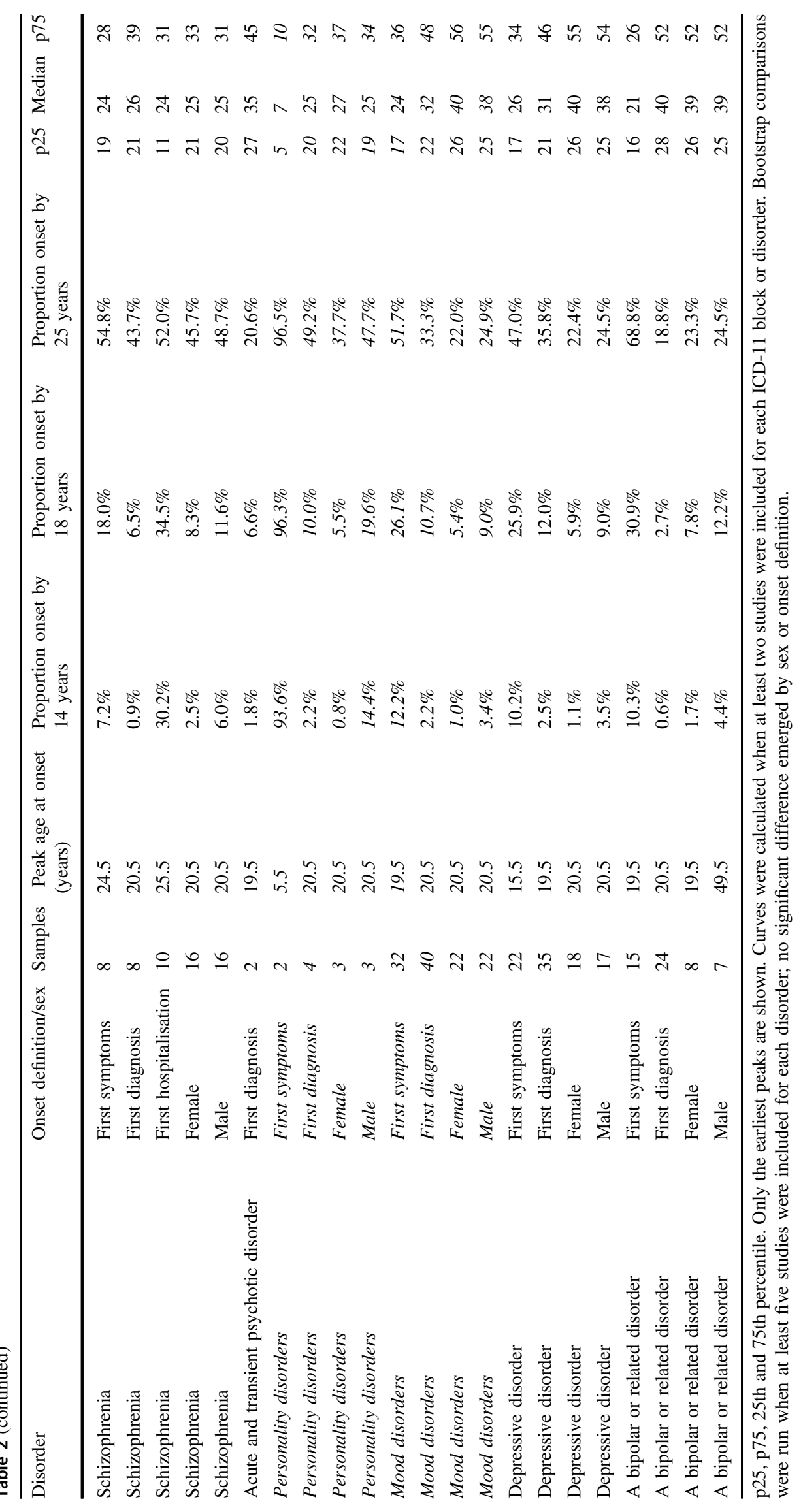


to account for differences across regions. Finally, caution is needed when comparing peaks (e.g., between symptoms and diagnosis), in particular when the peak curves are different, and when comparing median age at onset across disorders (e-Table 5).

\section{Conclusions}

The meta-analytic, global, epidemiological evidence provided challenges the current mental health care system that artificially separates child/adolescent and adult mental health services, providing strong epidemiologic evidence for the global implementation of integrated models of mental health promotion and preventive/early intervention care for young people in the community, those at risk for and with manifest mental disorders.

Acknowledgements This work was supported by Wellcome Trust Innovator Grant to PFP. Furthermore, the study was supported by Miguel Servet Research Contract (CPII19/00009 to JR), Alicia Koplowitz Foundation and Research Project Grants (PI19/00394) from the Instituto de Salud Carlos III and co-funded by European Union (ERDF/ESF, 'Investing in your future'). JBK is supported by the National Institute of Health Research University College London Hospital Biomedical Research Centre.

Author contributions PFP conceived the study, which was led by MS and JR. MO, EC, LS, GSdP extracted the data under the supervision of MS. MS, JR and PFP drafted the work, and JR conducted the analyses. JIS, JBK, PJ, CUC made substantial contributions to the interpretation of the results. All authors revised the manuscript critically for important intellectual content, approved the final version for publication and agreed to be accountable for all the aspects of the work. MS, JR had full access to all the data in the study and can take responsibility for the integrity of the data and the accuracy of the data analysis.

\section{Compliance with ethical standards}

Conflict of interest MS has been a consultant and/or advisor to or has received honoraria from Angelini, Lundbeck. CUC has been a consultant and/or advisor to or has received honoraria from Acadia, Alkermes, Allergan, Angelini, Axsome, Gedeon Richter, Gerson Lehrman Group, IntraCellular Therapies, Janssen/J\&J, LB Pharma, Lundbeck, MedAvante-ProPhase, Medscape, Neurocrine, Noven, Otsuka, Pfizer, Recordati, Rovi, Sumitomo Dainippon, Sunovion, Supernus, Takeda, and Teva. He has provided expert testimony for Janssen and Otsuka. He served on a Data Safety Monitoring Board for Lundbeck, Rovi, Supernus, and Teva. He received royalties from UpToDate and grant support from Janssen and Takeda. He is also a stock option holder of LB Pharma. PF-P has received grant fees from Lundbeck and honoraria from Lundbeck, Menarini and Angelini, outside the current work. All other authors declare no conflict of interest.

Publisher's note Springer Nature remains neutral with regard to jurisdictional claims in published maps and institutional affiliations.

Open Access This article is licensed under a Creative Commons Attribution 4.0 International License, which permits use, sharing, adaptation, distribution and reproduction in any medium or format, as long as you give appropriate credit to the original author(s) and the source, provide a link to the Creative Commons license, and indicate if changes were made. The images or other third party material in this article are included in the article's Creative Commons license, unless indicated otherwise in a credit line to the material. If material is not included in the article's Creative Commons license and your intended use is not permitted by statutory regulation or exceeds the permitted use, you will need to obtain permission directly from the copyright holder. To view a copy of this license, visit http://creativecommons. org/licenses/by/4.0/.

\section{References}

1. Hjorthøj C, Stürup AE, McGrath JJ, Nordentoft M. Years of potential life lost and life expectancy in schizophrenia: a systematic review and meta-analysis. Lancet Psychiatry. 2017;4:295-301.

2. Walker ER, McGee RE, Druss BG. Mortality in mental disorders and global disease burden implications a systematic review and meta-analysis. JAMA Psychiatry. 2015;72:334-41.

3. Saha S, Chant D, McGrath J. A systematic review of mortality in schizophrenia: Is the differential mortality gap worsening over time? Arch Gen Psychiatry. 2007;64:1123-31.

4. Nordentoft M, Wahlbeck K, Hällgren J, Westman J, Ösby U, Alinaghizadeh $\mathrm{H}$, et al. Excess mortality, causes of death and life expectancy in 270,770 patients with recent onset of mental disorders in Denmark, Finland and Sweden. PLoS ONE. 2013;8:e55176.

5. Fusar-Poli P, McGorry PD, Kane JM. Improving outcomes of first-episode psychosis: an overview. World Psychiatry. 2017;16:251-65.

6. Correll CU, Galling B, Pawar A, Krivko A, Bonetto C, Ruggeri $\mathrm{M}$, et al. Comparison of early intervention services vs treatment as usual for early-phase psychosis: a systematic review, meta-analysis, and meta-regression. JAMA Psychiatry. 2018;75:555-65.

7. Fusar-Poli P, Bauer M, Borgwardt S, Bechdolf A, Correll CU, Do $\mathrm{KQ}$, et al. European college of neuropsychopharmacology network on the prevention of mental disorders and mental health promotion (ECNP PMD-MHP). Eur Neuropsychopharmacol. 2019. https://doi.org/10.1016/j.euroneuro.2019.09.006.

8. Fusar-Poli P, Correll C, Arango C, Berk M, Patel V, Ioannidis J Preventive psychiatry: a blueprint for improving the mental health of young people. World Psychiatry. 2021;20:200-21.

9. Millan MJ, Andrieux A, Bartzokis G, Cadenhead K, Dazzan P, Fusar-Poli P, et al. Altering the course of schizophrenia: Progress and perspectives. Nat Rev Drug Discov. 2016;15:485-515.

10. Fusar-Poli P. The clinical high-risk state for psychosis (CHR-P), version II. Schizophr Bull. 2017;43:44-7.

11. Fusar-Poli P, Salazar de Pablo G, Correll CU, Meyer-Lindenberg A, Millan MJ, Borgwardt S, et al. Prevention of Psychosis: Advances in Detection, Prognosis, and Intervention. JAMA Psychiatry. 2020;77:755-65.

12. Catalan A, Salazar de Pablo G, Vaquerizo Serrano J, Mosillo P, Baldwin H, Fernández-Rivas A, et al. Annual research review: prevention of psychosis in adolescents-systematic review and meta-analysis of advances in detection, prognosis and intervention. J Child Psychol Psychiatry. 2020. https://doi.org/10.1111/ jcpp. 13322 .

13. Salazar de Pablo G, Catalan A, Fusar-Poli P. Clinical validity of DSM-5 attenuated psychosis syndrome: advances in diagnosis, prognosis, and treatment. JAMA Psychiatry. 2020;77:311-20.

14. Salazar de Pablo G, Radua J, Pereira J, Bonoldi I, Arienti V, Besana F, et al. Probability of transition to psychosis in individuals at clinical high risk: an updated meta-analysis. JAMA Psychiatry. 2021. (In press). 
15. Kotlicka-Antczak M, Podgórski M, Oliver D, Maric NP, Valmaggia L, Fusar-Poli P. Worldwide implementation of clinical services for the prevention of psychosis: the IEPA early intervention in mental health survey. Early Interv Psychiatry. 2020:eip.12950.

16. Fusar-Poli P, Byrne M, Badger S, Valmaggia LR, McGuire PK. Outreach and support in South London (OASIS), 2001-11: ten years of early diagnosis and treatment for young individuals at high clinical risk for psychosis. Eur Psychiatry. 2013;28:315-26.

17. Salazar de Pablo G, Estradé A, Cutroni M, Andlauer O, Fusar-Poli P. Establishing a clinical service to prevent psychosis: what, how and when? Systematic review. Transl Psychiatry. 2021;11:43.

18. Fusar-Poli P, Spencer T, De Micheli A, Curzi V, Nandha S, McGuire P. Outreach and support in South-London (OASIS) 2001-20: twenty years of early detection, prognosis and preventive care for young people at risk of psychosis. Eur Neuropsychopharmacol. 2020;39:111-22.

19. Fusar-Poli P, Davies C, Solmi M, Brondino N, De Micheli A, Kotlicka-Antczak M, Shin JI, Radua J. Preventive Treatments for Psychosis: Umbrella Review (Just the Evidence). Front Psychiatry. 2019;10:764.

20. Davies C, Cipriani A, Ioannidis JPA, Radua J, Stahl D, Provenzani $U$, et al. Lack of evidence to favor specific preventive interventions in psychosis: a network meta-analysis. World Psychiatry. 2018;17:196-209.

21. Bosnjak Kuharic D, Kekin I, Hew J, Rojnic Kuzman M, Puljak L. Interventions for prodromal stage of psychosis. Cochrane Database Syst Rev. 2019;11:CD012236.

22. Burke AS, Shapero BG, Pelletier-Baldelli A, Deng WY, Nyer $\mathrm{MB}$, Leathem L, et al. Rationale, methods, feasibility, and preliminary outcomes of a transdiagnostic prevention program for atrisk college students. Front Psychiatry. 2020;10:1030.

23. Radua J, Ramella-Cravaro V, Ioannidis JPA, Reichenberg A, Phiphopthatsanee N, Amir T, et al. What causes psychosis? An umbrella review of risk and protective factors. World Psychiatry. 2018;17:49-66.

24. Lindow JC, Hughes JL, South C, Minhajuddin A, Gutierrez L, Bannister E, et al. The youth aware of mental health intervention: impact on help seeking, mental health knowledge, and stigma in U.S. Adolescents. J Adolesc Health. 2020. https://doi.org/10. 1016/j.jadohealth.2020.01.006.

25. Salazar de Pablo G, De Micheli A, Nieman DH, Correll CU, Kessing LV, Pfennig A, et al. Universal and selective interventions to promote good mental health in young people: systematic review and meta-analysis. Eur Neuropsychopharmacol J Eur Coll Neuropsychopharmacol. 2020;41:28-39.

26. Fusar-Poli P, Salazar de Pablo G, De Micheli A, Nieman DH, Correll CU, Kessing LV, et al. What is good mental health? A scoping review. Eur Neuropsychopharmacol. 2020;31:33-46.

27. Xu L, Guo Y, Cao Q, Li X, Mei T, Ma Z, et al. Predictors of outcome in early onset schizophrenia: a 10-year follow-up study. BMC Psychiatry. 2020;20:67.

28. Chen L, Selvendra A, Stewart A, Castle D. Risk factors in early and late onset schizophrenia. Compr Psychiatry. 2018;80:155-62.

29. Fagerlund B, Pantelis C, Jepsen JRM, Raghava JM, Rostrup E, et al. Differential effects of age at illness onset on verbal memory functions in antipsychotic-naïve schizophrenia patients aged 12-43 years. Psychol Med. 2020:1-11.

30. Jones PB. Adult mental health disorders and their age at onset. $\mathrm{Br}$ J Psychiatry Suppl. 2013;54:s5-10.

31. Moher D, Liberati A, Tetzlaff J, Altman DG; PRISMA Group. Preferred reporting items for systematic reviews and meta-analyses: the PRISMA statement. PLoS Med. 2009;6:e1000097.

32. Stroup DF, Berlin JA, Morton SC, Olkin I, Williamson GD, Rennie D, et al. Meta-analysis of observational studies in epidemiology: a proposal for reporting. $\mathrm{J}$ Am Med Assoc. 2000;283:2008-12.
33. Wells GA, Shea B, O'Connell D, Peterson J, Welch V, Losos M, Tugwell P. Newcastle-Ottawa scale assess qual nonrandomised stud meta-analyses. Ottawa Hospital Research Institute. http://www.ohri.ca/ programs/clinical_epidemiology/oxford.asp. Accessed 21 Dec 2019.

34. Davison AC, Hinkley DV. Bootstrap methods and their application. Cambridge University Press; 1997.

35. Zaboski BA, Merritt OA, Schrack AP, Gayle C, Gonzalez M, Guerrero LA, et al. Hoarding: A meta-analysis of age of onset. Depress Anxiety. 2019;36:552-64.

36. Immonen J, Jääskeläinen E, Korpela H, Miettunen J. Age at onset and the outcomes of schizophrenia: a systematic review and metaanalysis. Early Interv. Psychiatry 2017;11:453-60.

37. Eranti SV, MacCabe JH, Bundy H, Murray RM. Gender difference in age at onset of schizophrenia: a meta-analysis. Psychol Med. 2013;43:155-67.

38. Myles N, Newall H, Compton MT, Curtis J, Nielssen O, Large M. The age at onset of psychosis and tobacco use: a systematic metaanalysis. Soc Psychiatry Psychiatr Epidemiol. 2012;47:1243-50.

39. Fusar-Poli P, Solmi M, Brondino N, Davies C, Chae C, Politi P, et al. Transdiagnostic psychiatry: a systematic review. World Psychiatry. 2019;18:192-207.

40. Fusar-Poli P. TRANSD recommendations: improving transdiagnostic research in psychiatry. World Psychiatry. 2019;18:361-2.

41. Paus T, Keshavan M, Giedd JN. Why do many psychiatric disorders emerge during adolescence? Nat Rev Neurosci. 2008;9:947-57.

42. Gałecki P, Talarowska M. Neurodevelopmental theory of depression. Prog Neuropsychopharmacol Biol Psychiatry. 2018;80:267-72.

43. Howes OD, Murray RM. Schizophrenia: an integrated sociodevelopmental-cognitive model. Lancet. 2014;383:1677-87.

44. Demjaha A, MacCabe JH, Murray RM. How genes and environmental factors determine the different neurodevelopmental trajectories of schizophrenia and bipolar disorder. Schizophr Bull. 2012;38:209-14.

45. Gore FM, Bloem PJN, Patton GC, Ferguson J, Joseph V, Coffey $\mathrm{C}$, et al. Global burden of disease in young people aged 10-24 years: a systematic analysis. Lancet. 2011;377:2093-102.

46. McGorry PD. Early intervention in psychosis: obvious, effective, overdue. J Nerv Ment Dis. 2015;203:310-8.

47. Bortolato B, Köhler CA, Evangelou E, León-Caballero J, Solmi M, Stubbs B, et al. Systematic assessment of environmental risk factors for bipolar disorder: an umbrella review of systematic reviews and meta-analyses. Bipolar Disord. 2017;19:84-96.

48. Kim JY, Son MJ, Son CY, Radua J, Eisenhut M, Gressier F, et al. Environmental risk factors and biomarkers for autism spectrum disorder: an umbrella review of the evidence. Lancet Psychiatry. 2019. 2019. https://doi.org/10.1016/S2215-0366(19)30181-6.

49. Köhler CA, Evangelou E, Stubbs B, Solmi M, Veronese N, Belbasis L, et al. Mapping risk factors for depression across the lifespan: An umbrella review of evidence from meta-analyses and Mendelian randomization studies. J Psychiatr Res. 2018;103:189-207.

50. Tortella-Feliu M, Fullana MA, Pérez-Vigil A, Torres X, Chamorro J, Littarelli SA, et al. Risk factors for posttraumatic stress disorder: an umbrella review of systematic reviews and metaanalyses. Neurosci Biobehav Rev. 2019;107:154-65.

51. Fullana MA, Tortella-Feliu M, Fernández de la Cruz L, Chamorro J, Pérez-Vigil A, Ioannidis JPA, et al. Risk and protective factors for anxiety and obsessive-compulsive disorders: an umbrella review of systematic reviews and meta-analyses. Psychol Med. 2020;50:1300-1315.

52. Belbasis L, Köhler CA, Stefanis N, Stubbs B, van Os J, Vieta E, et al. Risk factors and peripheral biomarkers for schizophrenia spectrum disorders: an umbrella review of meta-analyses. Acta Psychiatr Scand. 2018;137:88-97. 
53. Davies C, Segre G, Estradé A, Radua J, De Micheli A, Provenzani $\mathrm{U}$, et al. Prenatal and perinatal risk and protective factors for psychosis: a systematic review and meta-analysis. Lancet Psychiatry. 2020;7:399-410.

54. Arango C, Dragioti E, Solmi M, Cortese S, Domschke K, Murray R, et al. Evidence-based atlas of risk and protective factors of mental disorders: meta-umbrella review. World Psychiatry. 2021. (In press).

55. Werner-Seidler A, Perry Y, Calear AL, Newby JM, Christensen H. School-based depression and anxiety prevention programs for young people: a systematic review and meta-analysis. Clin Psychol Rev. 2017;51:30-47.

56. Lai ES, Kwok CL, Wong PW, Fu KW, Law YW, Yip PS. The Effectiveness and Sustainability of a Universal School-Based Programme for Preventing Depression in Chinese Adolescents: A Follow-Up Study Using Quasi-Experimental Design. PLoS ONE. 2016;26;11:e0149854.

57. Strøm HK, Adolfsen F, Handegård BH, Natvig H, Eisemann M, Martinussen $\mathrm{M}$, et al. Preventing alcohol use with a universal school-based intervention: results from an effectiveness study. BMC Public Health. 2015;15:337.

58. Fusar-Poli P. Integrated mental health services for the developmental period ( 0 to 25 years): a critical review of the evidence. Front Psychiatry. 2019;10:355.

59. McGorry PD, Hartmann JA, Spooner R, Nelson B. Beyond the "at risk mental state" concept: transitioning to transdiagnostic psychiatry. World Psychiatry. 2018;17:133-42.
60. Solmi M, Durbaba S, Ashworth M, Fusar-Poli P. Proportion of young people in the general population consulting general practitioners: potential for mental health screening and prevention. Early Interv Psychiatry. 2019. 2019. https://doi.org/10.1111/eip. 12908.

61. McGorry PD, Hartmann JA, Spooner R, Nelson B. Beyond the "at risk mental state" concept: transitioning to transdiagnostic psychiatry. World Psychiatry. 2018;17:133-42.

62. Tuomainen H, Schulze U, Warwick J, Paul M, Dieleman GC, Franić $\mathrm{T}$, et al. Managing the link and strengthening transition from child to adult mental health Care in Europe (MILESTONE): background, rationale and methodology. BMC Psychiatry. 2018;18:167. https://doi.org/10.1186/s12888-018-1758-z. Erratum in: BMC Psychiatry. 2018;18:295.

63. Russet F, Humbertclaude V, Dieleman G, Dodig-Ćurković K, Hendrickx G, Kovač V, et al. Training of adult psychiatrists and child and adolescent psychiatrists in Europe: a systematic review of training characteristics and transition from child/adolescent to adult mental health services. BMC Med Educ. 2019;19:204.

64. Leavey G, McGrellis S, Forbes T, Thampi A, Davidson G, Rosato $\mathrm{M}$, et al. Improving mental health pathways and care for adolescents in transition to adult services (IMPACT): a retrospective case note review of social and clinical determinants of transition. Soc Psychiatry Psychiatr Epidemiol. 2019;54:955-63.

\section{Affiliations}

\section{Marco Solmi (iD ${ }^{1,2,3} \cdot$ Joaquim Radua ${ }^{3,4,5} \cdot$ Miriam Olivola $^{3}$ Enrico Croce ${ }^{6} \cdot$ Livia Soardo $^{7}$. $^{7}$ Gonzalo Salazar de Pablo ${ }^{3,8,9} \cdot$ Jae II Shin ${ }^{10}$. James B. Kirkbride (iD ${ }^{11} \cdot$ Peter Jones (iD ${ }^{12,13} \cdot$ Jae Han Kim $^{14}$. Jong Yeob Kim ${ }^{14}$. Andrè F. Carvalho ${ }^{15}$ - Mary V. Seeman ${ }^{16}{ }^{16}$ Christoph U. Correll $^{17,18,19,20}$. Paolo Fusar-Poli (iD) $3,7,21,22$}

1 Neurosciences Department, University of Padua, Padua, Italy

2 Neuroscience Centre, University of Padua, Padua, Italy

3 Department of Psychosis Studies, Early Psychosis: Interventions and Clinical-detection (EPIC) Lab, Institute of Psychiatry, Psychology \& Neuroscience, King's College London, London, UK

4 Imaging Mood- and Anxiety-Related Disorders (IMARD) group, Institut d'Investigacions Biomèdiques August Pi i Sunyer (IDIBAPS), Mental Health Research Networking Center (CIBERSAM), Barcelona, Spain

5 Department of Clinical Neuroscience, Centre for Psychiatric Research and Education, Karolinska Institutet, Solna, Sweden

6 Department of Biomedical and Specialty Surgical Sciences, Institute of Psychiatry, University of Ferrara, Ferrara, Italy

7 Department of Brain and Behavioral Sciences, University of Pavia, Pavia, Italy

8 Department of Child and Adolescent Psychiatry, Institute of Psychiatry, Psychology \& Neuroscience, King's College London, London, UK

9 Department of Child and Adolescent Psychiatry, Institute of Psychiatry and Mental Health, Hospital General Universitario Gregorio Marañón School of Medicine, Universidad Complutense,
Instituto de Investigación Sanitaria Gregorio Marañón (IiSGM), CIBERSAM, Madrid, Spain

10 Department of Pediatrics, Yonsei University College of Medicine, Seoul, South Korea

11 Division of Psychiatry, University College London, London, UK

12 Department of Psychiatry, University of Cambridge, Cambridge, England

13 CAMEO Early Intervention Service, Cambridgeshire and Peterborough National Health Service Foundation Trust, Cambridge, England

14 Yonsei University College of Medicine, Seoul, South Korea

15 IMPACT (Innovation in Mental and Physical Health and Clinical Treatment) Strategic Research Centre, School of Medicine, Barwon Health, Deakin University, Geelong, VIC, Australia

16 Department of Psychiatry, University of Toronto, Toronto, ON, Canada

17 Department of Psychiatry, Zucker Hillside Hospital, Glen Oaks, NY, USA

18 Department of Psychiatry and Molecular Medicine, Donald and Barbara Zucker School of Medicine at Hofstra/Northwell, Hempstead, NY, USA 
19 Center for Psychiatric Neuroscience, Feinstein Institute for Medical Research, Manhasset, NY, USA

20 Department of Child and Adolescent Psychiatry, CharitéUniversitätsmedizin Berlin, Berlin, Germany
21 OASIS service, South London and Maudsley NHS Foundation Trust, London, UK

22 National Institute for Health Research, Maudsley Biomedical Research Centre, London, UK 